\title{
LETTER
}

\section{Stem cell biology}

\section{FAM122A is required for hematopoietic stem cell function}

\author{
Man-Hua Liu $\mathbb{D}^{1} \cdot$ Xiao-Cui Zhang $\mathbb{D}^{1} \cdot$ Jing Chen ${ }^{1} \cdot$ Yun-Sheng Yang ${ }^{1} \cdot$ Yin-Qi Wang ${ }^{1} \cdot$ Jun-Ke Zheng ${ }^{1} \cdot$ \\ Guo-Qiang Chen $\mathbb{1}^{1} \cdot$ Ying Huang $\mathbb{1}^{1}$
}

Received: 16 June 2020 / Revised: 14 October 2020 / Accepted: 15 November 2020 / Published online: 1 December 2020

(c) Springer Nature Limited 2020

\section{To the Editor:}

The homeostasis of hematopoietic stem cell (HSC) is critical for maintaining whole blood system throughout the entire organism life span, in which HSCs keep the balance of quiescence, self-renewal, differentiation, and apoptosis through a tight regulation of intrinsic and extrinsic signaling pathways [1]. More than $70 \% \mathrm{HSCs}$ are in the quiescent $\mathrm{G}_{0}$ stage, since a dormant status is necessary to preserve their self-renewal capacities and prevent stem cell exhaustion $[2,3]$. In response to blood cell loss or other stress stimulation, HSCs may rapidly enter cycling and replenish the hematopoietic system [4].

Family with sequence similarity $122 A$ (FAM122A) is a human housekeeping gene, localized on chromosome 9q21.1 within the first intron of PIP5K1B (phosphatidylinositol 4-phosphate 5-kinase) gene. It has 287 amino acids with highly conserved in many species of mammary animals. Previously we reported that FAM122A is an inhibitor of protein phosphatase 2A [5], and more recently, FAM122A has been found to be abnormally upregulated in acute myeloid leukemia (AML) characterized with the dysfunction of myeloid stem cells [6], and is essential for maintaining the growth of AML cells in vitro cultured cells and in vivo AML

Supplementary information The online version of this article (https:// doi.org/10.1038/s41375-020-01099-9) contains supplementary material, which is available to authorized users.

Guo-Qiang Chen

chengq@shsmu.edu.cn

$\bowtie$ Ying Huang

huangying@shsmu.edu.cn

1 Department of Pathophysiology, Key Laboratory of Cell Differentiation and Apoptosis of Chinese Ministry of Education and Chinese Academy of Medical Sciences Research Unit (NO.2019RU043), Rui-Jin Hospital, Shanghai Jiao Tong University School of Medicine (SJTU-SM), Shanghai, China cell engraftment [7]. In this study, we investigated whether FAM122A has a role in the function of HSCs.

By examining Gene Expression Common database of hematopoietic cells [8], mouse Fam122a gene is ubiquitously expressed in HSC and committed cells (supplemental Fig. 1A), which was confirmed by qPCR (Supplementary Fig. 1B). To further investigate the contribution of FAM122A in HSC biology, Fam 122 $a^{\text {flox/flox }}$ mice (supplemental Fig. 1C) were crossed with Vav-iCre transgenic mice [9] to generate the mice where Fam122a is genetically inactivated early in the development within hematopoietic and endothelial cells. Analysis of the genomic DNAs and proteins isolated from bone marrow (BM) demonstrated that FAM122A protein, rather than PIP5K1B, was efficiently deleted in Fam $122 a^{\text {flox/flox }}$ VaviCre mice (Fig. 1A and Supplementary Fig. 1D), hereafter designating Fam $122 a^{\text {flox/flox }}$ Vav-iCre as Fam $122 a^{\mathrm{CKO}}$ and Fam $122 a^{+1+}$ Vav-iCre as Fam $122 a^{\text {WT }}$. Animals of all genotypes were born as expected frequencies (data not shown) and seemed normal and fertile. Intriguingly, we found that Fam122a depletion resulted in a significant decrease in the frequencies of T lymphocytes $\left(\mathrm{CD}^{+}\right)$and $\mathrm{NK}$ cells $\left(\mathrm{NK} 1.1^{+} / \mathrm{CD}^{-}\right)$in peripheral bloods $(\mathrm{PBs})$ (Fig. 1B and Supplementary Fig. 1E), indicating that the differentiations of $\mathrm{T}$ and $\mathrm{NK}$ cells were compromised. Consistently, the frequencies of two subtypes of $\mathrm{T}$ lymphocytes $\left(\mathrm{CD}^{+} \mathrm{T}\right.$ helper cells and $\mathrm{CD}^{+}$cytotoxic $\mathrm{T}$ cells) were also reduced in $\mathrm{PBs}$ of Fam $122 a^{\mathrm{CKO}}$ mice, whereas FAM122A deletion did not influence the frequencies of B cells $\left(\mathrm{B} 220^{+}\right)$and dendritic cells $\left(\mathrm{MHCll}^{+} /\right.$ CD11 $\mathrm{c}^{+}$).

The total BM cell numbers were not significantly changed upon Fam122a deletion (Supplementary Fig. 1F), however, FACS analysis showed that Fam $122 a^{\mathrm{CKO}}$ mice had a higher frequency of long-term HSCs (LT-HSCs, CD34 ${ }^{-}$CD135 LSK) [10] in the BMs, while the frequencies of short-term HSCs (ST-HSCs, CD34 ${ }^{+}$CD135 ${ }^{-}$LSK) [10] and multipotent progenitors (MPPs, CD $34^{+} \mathrm{CD} 135^{+} \mathrm{LSK}$ ) demonstrated to be prone to decrease and significantly decreased, compared with 
A

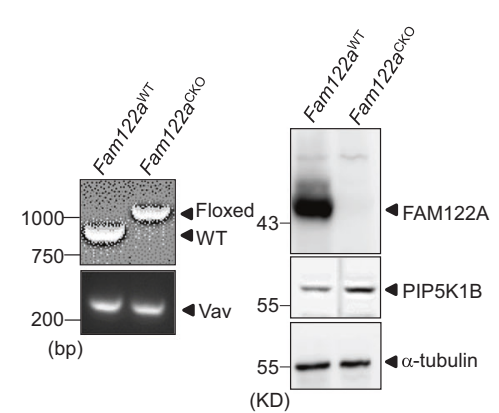

C

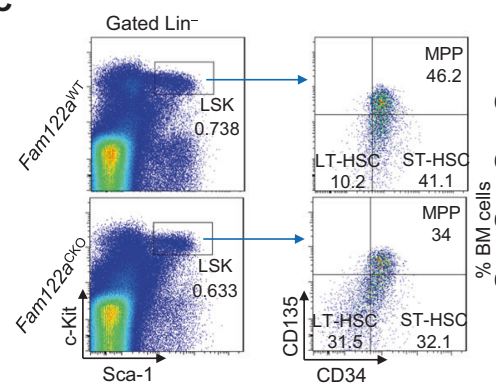

E

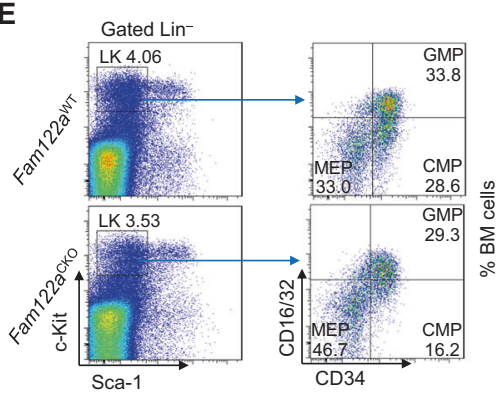

B

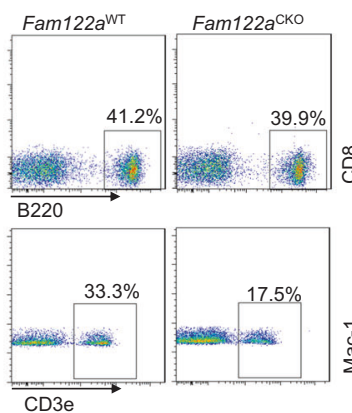

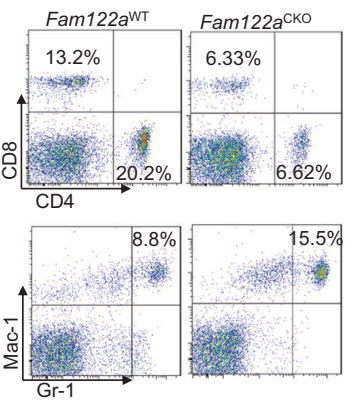

D
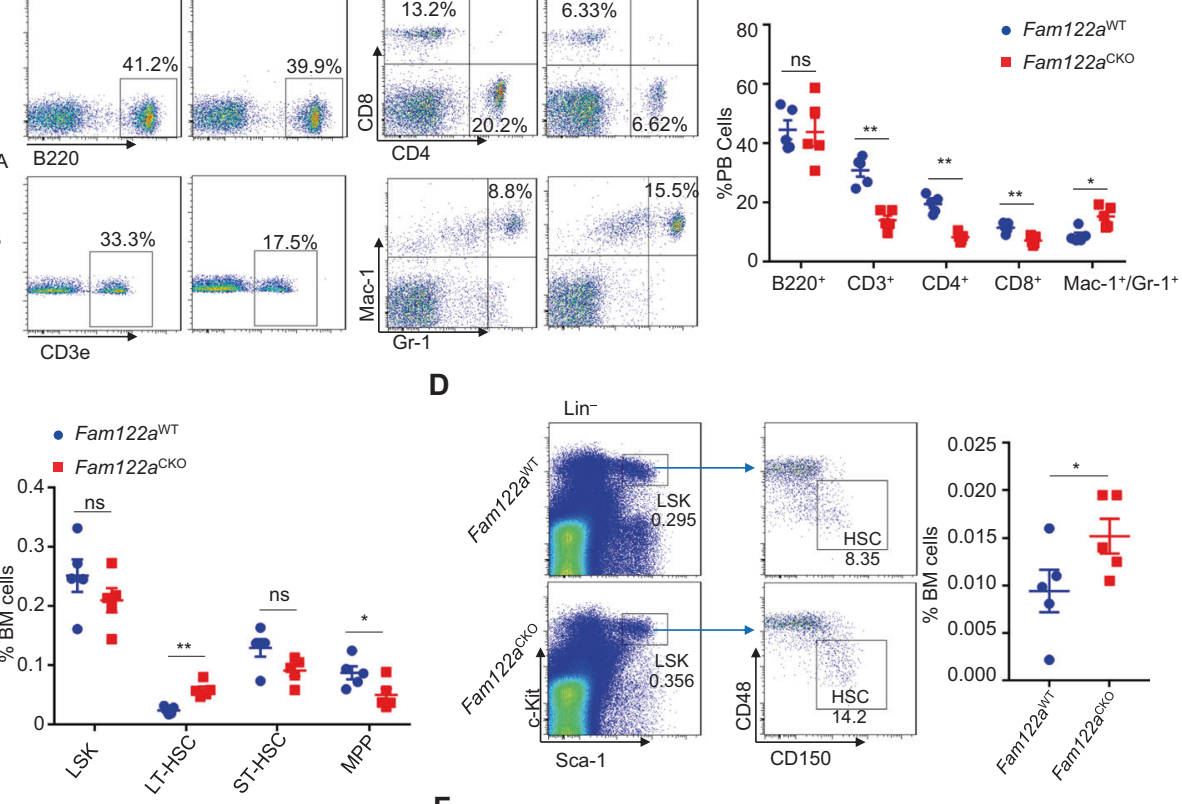

$\mathbf{F}$
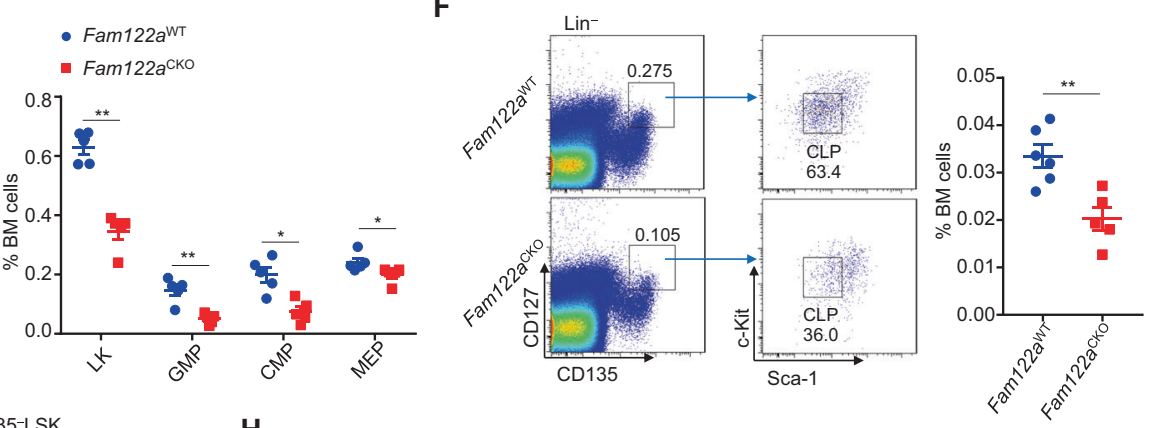

G
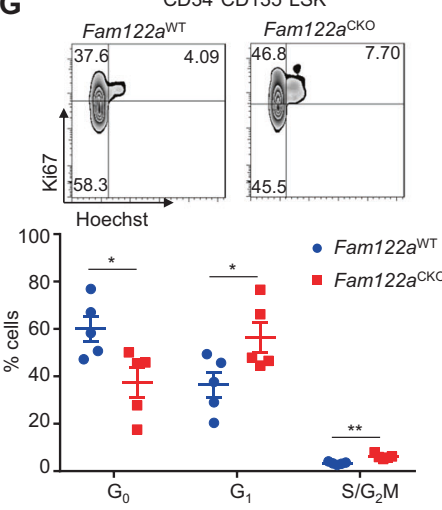

H
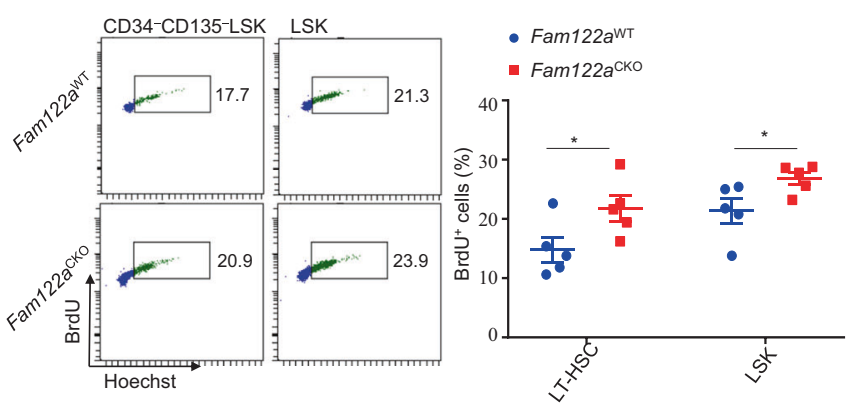

those of Fam122a $a^{\text {WT }}$ (Fig. 1C). The enhanced primitive HSC population was further confirmed by another stringent set of HSC markers [11], CD150 and CD48 (CD150 ${ }^{+} \mathrm{CD} 48^{-}$LSK) (Fig. 1D). FAM122A deletion also led to the reduced frequencies of committed progenitor cells LK $\left(\mathrm{Lin}^{-} \mathrm{c}-\mathrm{kit}^{+}\right)$, granulocyte/monocyte progenitors (GMPs, CD $34^{+} \mathrm{CD} 16 / 32^{+}$ $\mathrm{LK}$ ), common myeloid progenitors (CMPs, $\mathrm{CD} 34^{+} \mathrm{CD} 16 / 32^{-}$ LK), megakaryocyte/erythrocyte progenitors (MEPs, CD34 ${ }^{-}$ CD16/32- LK) and common lymphoid progenitors (CLPs,
$\mathrm{Lin}^{-} \mathrm{Sca}-1^{\text {low }} \mathrm{c}-\mathrm{Kit}^{\text {low }} \mathrm{CD} 127^{+} \mathrm{CD} 135^{+}$) (Fig. 1E, F). Further, we conducted cell cycle and cell proliferation analysis on LTHSCs (CD34 ${ }^{-} \mathrm{CD} 135^{-}$LSK). Ki67 staining revealed a significantly higher percentage of cycling cells $\left(\mathrm{Ki} 7^{+}\right)$accompanied with the decrease in $\mathrm{G}_{0}$ quiescent cells in Fam122a $a^{\mathrm{CKO}}$ LT-LSC cells (Fig. 1G). Pulse bromodeoxyuridine (Brdu) labeling further showed the relatively higher percentages of proliferating cells in Fam $122 a^{\mathrm{CKO}}$ LT-LSC and HSPC cells (Fig. 1H), while there is no change in apoptotic states 
4 Fig. 1 Fam122a deletion promotes quiescent LT-HSC into cycling. A Genotyping of Fam 122a ${ }^{\text {WT }}$ and Fam $122 a^{\mathrm{CKO}}$ mice (left). Knockout efficiency of FAM122A was confirmed in the BMs of indicated mice by western blot (right). B Representative FACS plots (left) and quantification of the frequencies (right) of peripheral blood lineages $\left(n=5\right.$ mice). $\mathrm{B} 220^{+}: \mathrm{B}$ cells; $\mathrm{CD}^{+}$: $\mathrm{T}$ cells; $\mathrm{CD}^{+}$: helper $\mathrm{T}$ cells; $\mathrm{CD}^{+}$: toxic T cells; $\mathrm{Mac}-1^{+} / \mathrm{Gr}-1^{+}$: myeloid cells. C Representative FACS plots (left) and quantification (right) of the frequencies of LSK $\left(\mathrm{Lin}^{-} \mathrm{Sca}-{ }^{+} \mathrm{c}-\mathrm{Kit}^{+}\right)$cells, LT-HSCs, ST-HSCs, and MPPs from Fam122a ${ }^{\mathrm{WT}}$ and Fam122a ${ }^{\mathrm{CKO}}$ BMs. LT-HSCs long-term HSCs, STHSCs short-term HSCs, MPPs pluripotent hematopoietic progenitor cells $(n=5$ mice). D Representative FACS plots and quantification of frequency of LT-HSCs ( $\mathrm{Lin}^{-} \mathrm{Sca}-1^{+} \mathrm{c}-\mathrm{Kit}^{+} \mathrm{CD} 150^{\text {high }} \mathrm{CD} 48^{\text {low }}$ ) from indicated mice ( $n=5$ mice). Representative FACS plots and quantification of the frequencies of LK $\left(\mathrm{Lin}^{-} \mathrm{c}-\mathrm{Kit}^{+}\right.$) cells, GMPs, CMPs, and MEPs $(\mathbf{E})(n=5$ mice $)$; or CLPs $(\mathbf{F})$ from BMs in Fam $122 a^{\text {WT }}$ and Fam122a ${ }^{\mathrm{CKO}}$ mice $\left(n=6\right.$ mice for Fam $122 a^{\mathrm{WT}}, n=5$ mice for Fam122a ${ }^{\mathrm{CKO}}$ ). GMPs granulocyte-monocyte progenitor cells, CMPs common myeloid progenitor cells, MEPs megakaryocyte-erythroid progenitor cells, CLPs common lymphoid precursor cells. G Cellcycle analysis of Fam122a WT and Fam122a ${ }^{\text {CKO }}$ LT-HSCs (CD34 ${ }^{-}$ CD135 ${ }^{-}$LSK). FACS plots for Ki67 and Hoechst 33342 staining (upper) and statistical analysis of $\mathrm{G}_{0}, \mathrm{G}_{1}, \mathrm{~S} / \mathrm{G}_{2} / \mathrm{M}$ (bottom) $(n=5$ mice). $\mathbf{H}$ Proliferation assay of the LT-HSCs (CD34 $\left.{ }^{-} \mathrm{CD} 135^{-} \mathrm{LSK}\right)$ and LSK cells in Fam122a ${ }^{\text {WT }}$ and Fam122a ${ }^{\mathrm{CKO}}$. Representative FACS plots for BrdU and Hoechst 33342 staining (left) and the statistical analysis of BrdU positive cells (right) $(n=5$ mice). All data are presented as mean \pm SEM from an independent experiment. ${ }^{*} p<0.05$; $* * p<0.01 ;$ ns no significance.

upon FAM122A deletion with Annexin-V staining (Supplementary Fig. 2).

To test whether there are functional defects in Fam122anull HSCs, we performed competitive BM transplantation experiments as indicated in Fig. 2A, and found that the repopulation activity of Fam $122 a^{\mathrm{CKO}}$ BMs was dramatically decreased compared with Fam122a $a^{\text {WT }}$ cells (left, Fig. 2B, C). Furthermore, Fam122a-null donor cells displayed an impaired ability to repopulate $\mathrm{CD}^{+}$and $\mathrm{Mac}-1^{+} /$ Gr- $1^{+}$cells in PBs after transplantation, indicating the differentiations of $\mathrm{T}$ lymphocytes and granulocytes were affected in Fam122a-null cells post-transplantation (Fig. 2D). The analysis of recipient mouse BMs or PBs (day 20 post-transplantation) further revealed remarkable defects in the multi-lineage constitution of Fam122a-deficient donor-derived cells (Supplementary Fig. 3). A secondary transplantation with the same numbers of primary recipient mouse BMs displayed a similar trend in repopulation (right, Fig. 2B), suggesting Fam122a deletion leads to the loss of the self-renewal capability of HSCs. Consistently, Fam122a-null LT-HSCs expanded much more slowly when they were subjected to in vitro culturing (Fig. 2E). We did not detect any defects in the homing efficiency of HSCs upon Fam122a depletion (Supplementary Fig. 4). To substantiate the role of FAM122A in HSC

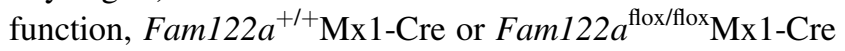
BMs were first transplanted into lethally irradiated mice without inducing Fam122a deletion. These recipient mice were then injected with $\mathrm{pIpC}$ at 8 weeks after transplantation to induce Fam122a deletion, followed by PB chimerism analysis. Consistent with the effect of Fam $122 a^{\mathrm{CKO}}$, Fam 122 $a^{\text {flox/flox } M x 1-C r e ~ B M s ~ e x h i b i t e d ~ a ~ p r o f o u n d ~ f a i l u r e ~}$ in the blood reconstitution (Fig. 2F). Collectively, FAM122A deletion led to a higher frequency of LT-HSCs with abnormally rapid proliferation capacity (decreasing $\mathrm{G}_{0}$ quiescent cells and increasing $\mathrm{Ki}^{+}$cells), moreover Fam122a deletion demonstrates a reduction in in vivo repopulating stem cell activity, suggesting FAM122A is essential for maintaining the quiescence and function of HSCs. On the other hand, the reduced frequencies of STHSCs, MPPs, and committed progenitors in Fam $122 a^{\mathrm{CKO}}$ mice indicate that the differentiation process is also compromised from LT-HSCs upon Fam 122a deletion.

To explore the mechanism of FAM122A maintaining HSC function, we performed RNA sequencing using LTHSCs derived from Fam 122a $a^{\text {WT }}$ and Fam 122 $a^{\text {CKO }}$ BMs (Fig. 2G). The overlapping regulation genes from two biological replication were 4582, in which 1312 were upregulated and 3270 were downregulated (Fig. 2H). KEGG analysis showed that most of these regulatory genes were enriched in PI3K-AKT, Rap-1, MAPK, and calcium signaling et al. (Fig. 2I). With the application of capillary electrophoresis, we further found that FAM122A deletion significantly enhanced phosphorylated p38, but not phosphorylated and total AKT, ERK, and MYC proteins (these three proteins are PP2A substrates) as well as total p38 (Fig. 2J, K and Supplementary Fig. 5). RNA-seq data further showed that most upregulated genes in MAPK signaling upon Fam 122a deletion were mainly involved in p38 MAPK pathway, which was further confirmed by qPCR in partial genes including the upregulation of Daxx, Mapk11 [12], and Tabl, and the downregulation of its negative regulator Ptprr (Fig. 2L). Evidence shows that the inactivation of p38 MAPK signaling is essential for maintaining HSC quiescence and its repopulating capacity [13]. Thus, our data support the notion that FAM122A deletion promotes LT-HSCs from quiescence to cycling through the activation of p38 MAPK signaling.

Deeply and widely understanding the regulation network of maintaining normal HSC function is essential for comprehending stem cell physiology and developing stem cell therapy such as stem cell transplantation, thus establishing appropriate therapeutic strategies for those patients suffered from leukemia and anemia [14]. In this study, we found that the deletion of Fam122a, a conserved housekeeping gene, leads to the loss of HSC functions including disrupting quiescent states, skewing HSPC differentiation, and impairing $\mathrm{BM}$ reconstitution capabilities. Moreover, we also found that FAM122A silencing could significantly suppress the growth of leukemia stem cells (LSCs) in the BM cells from AML patients (Supplementary Fig. 6), 
A

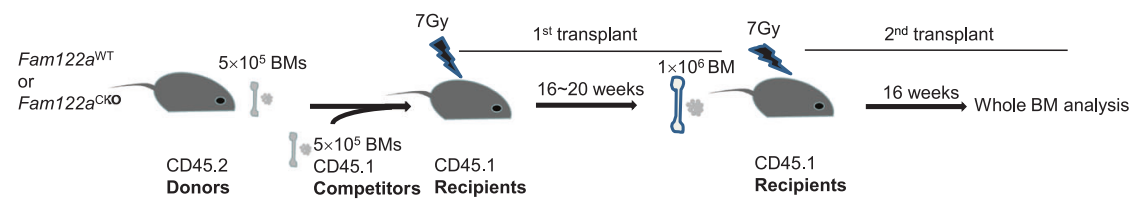

B
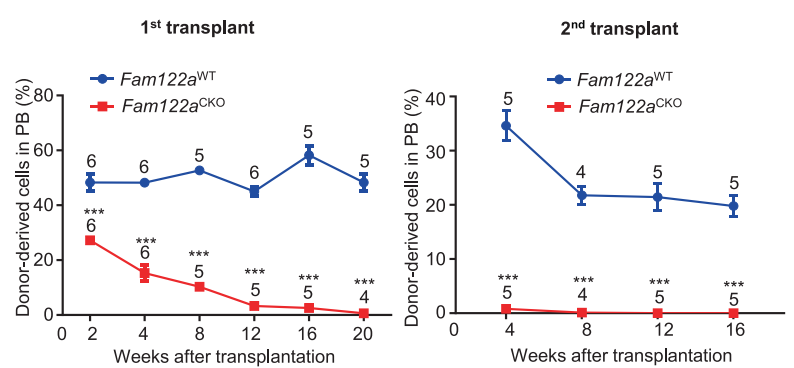

C

20 weeks in $1^{\text {st }}$ transplant

D
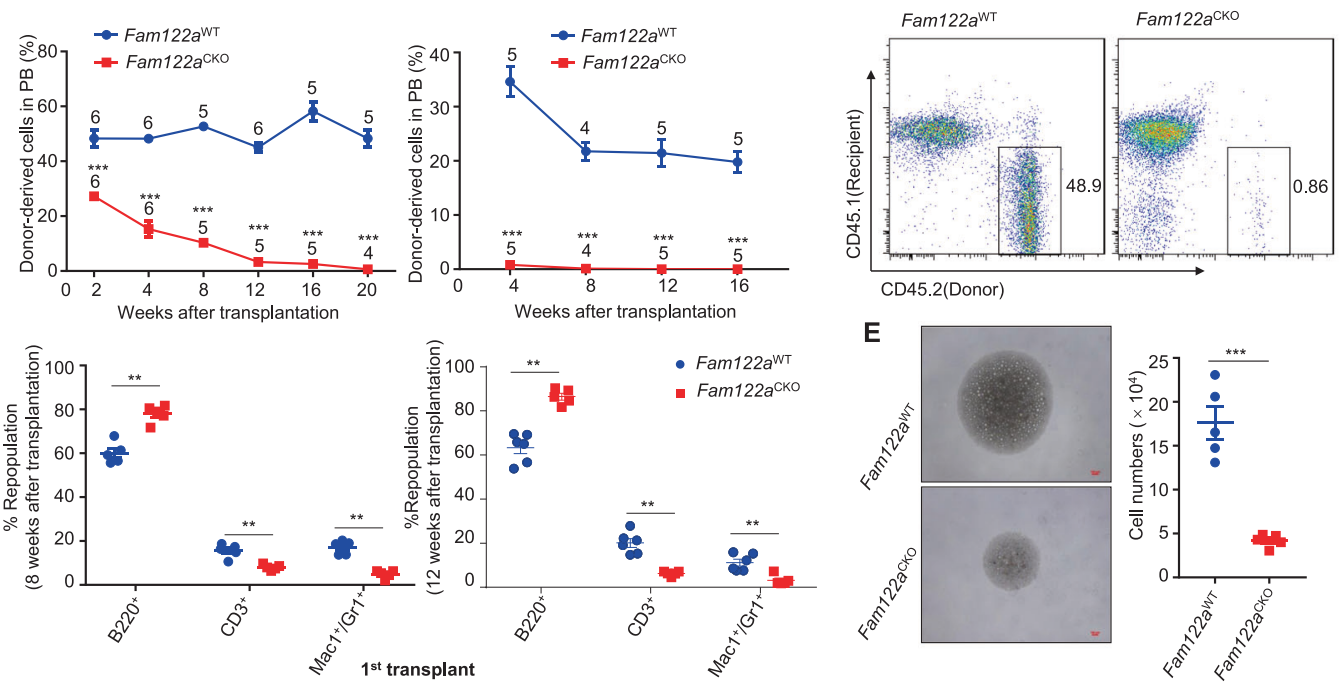

$\mathbf{F}$

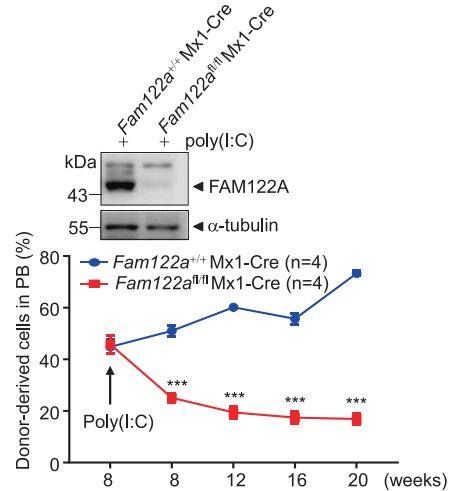

G

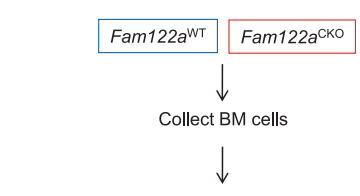

Sorting LT-HSCs pooled from 3 animals (2 Replicates)

CDNA amplification (SMART-Seq $\circledast$ V4 Ultra® Low Input RNA Kit for Sequencing)
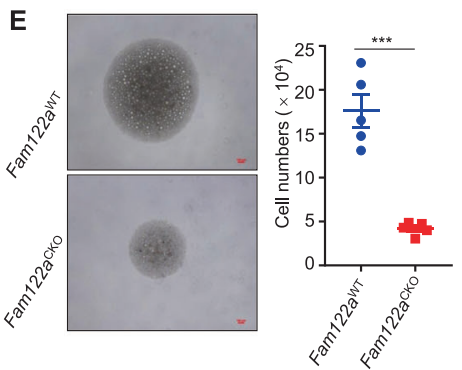

H
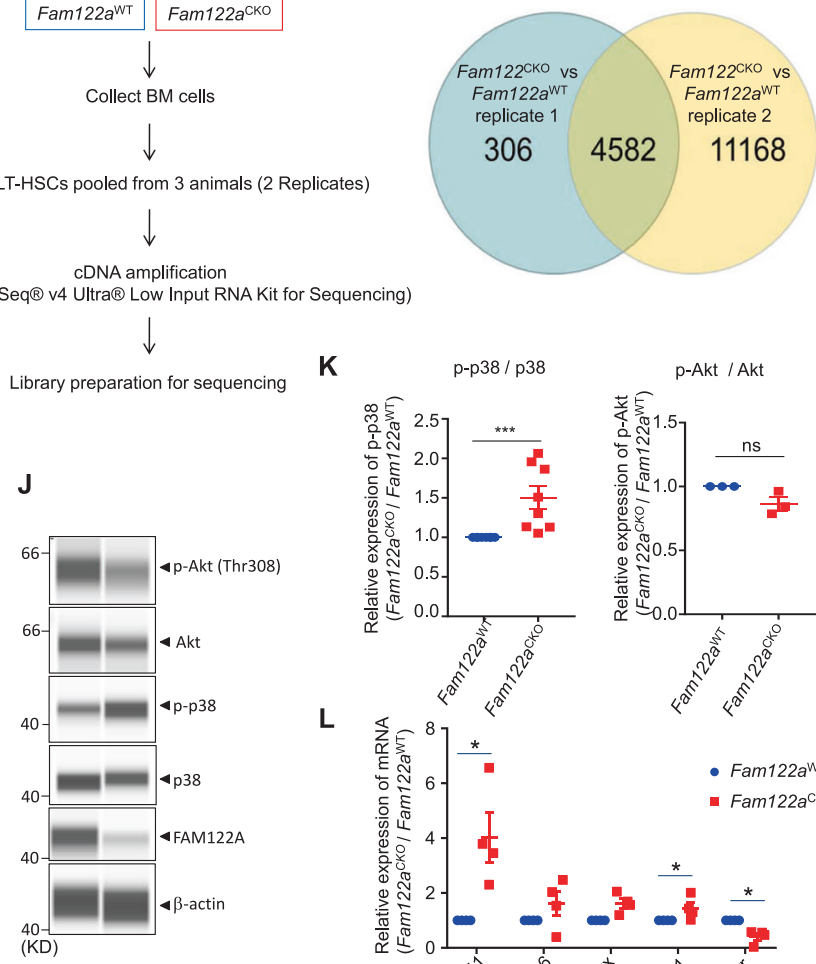

K
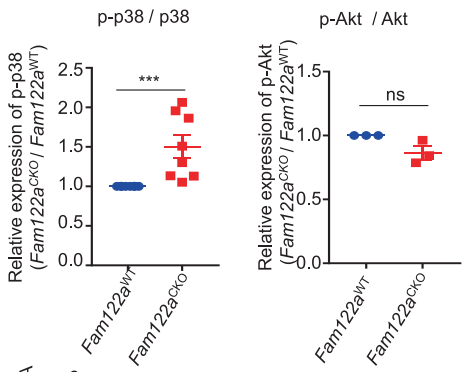

$\mathbf{L}$

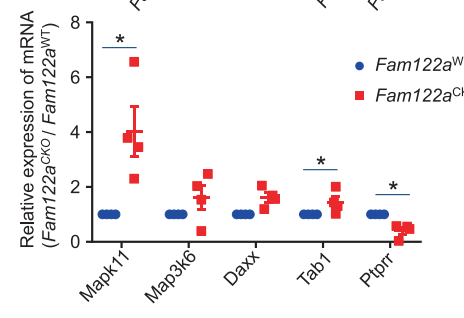

supporting the important role of FAM122A involved in mediating HSC functions. Further study showed that the loss of HSC function upon FAM122A deletion results in enhanced p38 MAPK signaling activation, rather than affecting PP2A activity (the phosphorylation of its substrates including AKT, ERK, and MYC) or the expression 
Fig. 2 Fam122a deletion compromises the long-term repopulation capacity of HSCs. A Schematics for serial competitive transplantation assay with Fam $122 a^{\text {WT }}$ and Fam $122 a^{\mathrm{CKO}}$ BM cells. B Statistical analysis of the ratio of donor cells in peripheral bloods (PBs) of Fam122a ${ }^{\mathrm{WT}}$ and Fam $122 a^{\mathrm{CKO}}$ recipient mice at different time points in a serial competitive BM transplantation ( $n=4-6$ mice per group as indicated). C Representative FACS plots for the proportion of donor cells in PBs of Fam 122a $a^{\mathrm{WT}}$ and Fam122a $a^{\mathrm{CKO}}$ recipient mice at 20 weeks posttransplantation. D Statistical analysis of Fam122a ${ }^{\mathrm{WT}}$ and Fam122a $a^{\mathrm{CKO}}$ donor B cells $\left(\mathrm{B} 220^{+}\right)$, T cells $\left(\mathrm{CD}^{+}\right)$and myeloid cells $\left(\mathrm{Mac}-1^{+} / \mathrm{Gr}-1^{+}\right)$ in PBs of recipient mice at 8 and 12 weeks post transplantation $(n=5$ mice). E Representative images of LT-HSCs from indicated mice culturing within basic medium (SCF + TPO) in vitro for 10 days (left). Cell numbers were evaluated (right) ( $n=5$ mice). F FAM122A deletion was confirmed in Fam122 $a^{\text {flox/flox }}$ Mx1-Cre BM cells, which was induced with poly(I:C) treatment post transplantation for 8 weeks (upper). Subsequently, the percentages of donor-derived PB cells were analyzed at indicated time points in competitive transplantation assay (bottom) $(n=4$ mice). G RNA-seq workflow. H Venn diagram showed 4582 genes coregulated in the replicate LT-HSC samples as performed in $(\mathbf{G})$ with fold change $\geq 2$. I KEGG analysis of the regulating genes enriched in the indicated signaling pathways. J, K Jess-based western blot was performed to examine the indicated proteins from LT-HSCs of Fam $122 a^{\text {WT }}$ and Fam $122 a^{\mathrm{CKO}}$ (the cells were from 3 mice per group). The representative images were shown $(\mathbf{J})$, and the relative p-p38 and p-Akt protein levels were analyzed against total $\mathrm{p} 38$ or AKT and normalized by those of Fam122a WT LT-HSCs (K). L The expression of the genes involved in p38 MAPK signaling pathway were examined by qPCR $(n=4$ mice $)$. All data are presented as mean \pm SEM from an independent experiment. ${ }^{*} p<0.05 ; * * p<0.01 ; * * * p<0.001$; ns no significance.

of PP2A subunits (Supplementary Fig. 5). Previously we have demonstrated that FAM122A silencing suppresses the growth of AML cells through modulating PP2A activity and MYC expression [7]. These combined results imply that the suppressing effects of FAM122A deletion on the growth of HSCs and LSCs or AML cells may involve the different signaling pathways, or the discrepancy existed in human and mouse hematological cells. Actually, we still did not know the exact reason for how FAM122A regulates p38 MAPK signaling until now. Increasing evidence show ROS level, upstream kinase activity and phosphotase are all important factors for modulating p38 activity [15]. Whether FAM122A may influence ROS level in HSCs and/or interact with the kinases or other phosphatases essential for modulating p38 MAPK signaling deserves to be investigated in future. In summary, our study provides first demonstration that FAM122A is essential for maintaining the quiescence and function of HSCs possibly by suppressing p38 MAPK signaling.

Acknowledgements This study was supported by the grants from Chinese National Natural Science Foundation $(81572290$ and 32070720 from YH; 81830091, 91853206 and 81972583 from GQC) and its innovative group support (81721004 from GQC),
CAMS Innovation Fund for Medical Sciences (CIFMS) (2019-I2M-5051) as well as the Fundamental Research Funds for the Central Universities.

\section{Compliance with ethical standards}

Conflict of interest All authors declare that they have no conflict of interest.

Publisher's note Springer Nature remains neutral with regard to jurisdictional claims in published maps and institutional affiliations.

\section{References}

1. Zon LI. Intrinsic and extrinsic control of haematopoietic stem-cell self-renewal. Nature. 2008;453:306-13.

2. Wang Z, Ema H. Mechanisms of self-renewal in hematopoietic stem cells. Int J Hematol. 2016;103:498-509.

3. Tumpel S, Rudolph KL. Quiescence: good and bad of stem cell aging. Trends Cell Biol. 2019;29:672-85.

4. Trumpp A, Essers M, Wilson A. Awakening dormant haematopoietic stem cells. Nat Rev Immunol. 2010;10:201-9.

5. Fan L, Liu MH, Guo M, Hu CX, Yan ZW, Chen J, et al. FAM122A, a new endogenous inhibitor of protein phosphatase 2A. Oncotarget. 2016;7:63887-900.

6. De Kouchkovsky I, Abdul-Hay M. 'Acute myeloid leukemia: a comprehensive review and 2016 update'. Blood Cancer J. 2016;6:e441.

7. Liu MH, Chen J, Yang YS, Wang YQ, Chen GQ, Zhang Y, et al. FAM122A promotes acute myeloid leukemia cell growth through inhibiting PP2A activity and sustaining MYC expression. Haematologica. 2020. https://doi.org/10.3324/haematol.2020.251462.

8. Bagger FO, Rapin N, Theilgaard-Monch K, Kaczkowski B, Jendholm J, Winther O, et al. HemaExplorer: a Web server for easy and fast visualization of gene expression in normal and malignant hematopoiesis. Blood. 2012;119:6394-5.

9. Georgiades P, Ogilvy S, Duval H, Licence DR, Charnock-Jones DS, Smith SK, et al. VavCre transgenic mice: a tool for mutagenesis in hematopoietic and endothelial lineages. Genesis. 2002; 34:251-6.

10. Christensen JL, Weissman IL. Flk-2 is a marker in hematopoietic stem cell differentiation: a simple method to isolate long-term stem cells. Proc Natl Acad Sci USA. 2001;98:14541-6.

11. Kiel MJ, Yilmaz OH, Iwashita T, Yilmaz OH, Terhorst C, Morrison SJ. SLAM family receptors distinguish hematopoietic stem and progenitor cells and reveal endothelial niches for stem cells. Cell. 2005;121:1109-21.

12. Tesio M, Tang Y, Mudder K, Saini M, von Paleske L, Macintyre $\mathrm{E}$, et al. Hematopoietic stem cell quiescence and function are controlled by the CYLD-TRAF2-p38MAPK pathway. J Exp Med. 2015;212:525-38.

13. Ito K, Hirao A, Arai F, Takubo K, Matsuoka S, Miyamoto K, et al. Reactive oxygen species act through p38 MAPK to limit the lifespan of hematopoietic stem cells. Nat Med. 2006;12: 446-51.

14. Zakrzewski W, Dobrzynski M, Szymonowicz M, Rybak Z. Stem cells: past, present, and future. Stem Cell Res Ther. 2019;10:68.

15. Zarubin T, Han J. Activation and signaling of the p38 MAP kinase pathway. Cell Res. 2005;15:11-8. 